\title{
Ultraschall 2020: Quo vadis?
}

\author{
Was gibt es Neues und Spannendes in der Sonografie \\ in diesem bewegten «Corona-Jahr»?
}

Wenn wir auf die Entwicklung der Sonografie zurückblicken, dann wurde vieles, was früher behauptet, jedoch nicht immer verstanden wurde, realisiert. In den späten 1970er Jahren hat Rettenmaier einen entscheidenden Paradigmawechsel erreicht: «Die Ultraschalldiagnostik ist die Fortsetzung der körperlichen Untersuchung mit technischen Mitteln»; er hat es auch als «Stethoskop des Abdomens» bezeichnet [1]. Also kein «bildgebendes Verfahren», wie man es später zusammen mit CT und MRT in der Radiologie zusammenfasste.

Mit den neuen Techniken, in den 1990er Jahren FKDS und PWDS, nach 2000 auch Elastografie und CEUS, haben sich neue Horizonte eröffnet. Kürzlich haben wir die Anwendung von CEUS bei fokalen Nierenveränderungen in «Praxis» publiziert [2], und in dieser Ausgabe erscheint auch eine exzellente Arbeit über die Anwendung von CEUS am Darm [3]. Obwohl man zuerst über die ThoraxSonografie meinte, da sei nur Luft, da sieht man nichts, können heute erstaunliche Möglichkeiten der Thoraxsonografie [4] vorgestellt werden.

Die Techniken an der Spitze der Entwicklung liessen jedoch die Anwendung bei vielen Nutzerinnen und Nutzern weit hinter sich. Heute hat praktisch jedes Ultraschallgerät einen Farbdoppler, er wird jedoch eher zu selten angewendet. Der Beitrag über Artefakte der Farbdopplersonografie [5] sollte hier kleine Abhilfe leisten. Die Qualität der medizinischen Versorgung entscheidet sich nicht in hochspezialisierten Zentren, sondern in erster Linie bei Hausärztinnen und -ärzten [6]. Die SGUM war bereits seit 1990 intensiv bemüht, gute Weiterbildungskurse für Ärzte zu entwickeln, 2000 wurde der Fähigkeitsausweis Sonografie geboren. In den folgenden Jahren kam es zur Harmonisierung dieser Kurse zwischen der Schweiz, Deutschland und Österreich, was die Möglichkeiten der Weiterbildung sinnvoll erweiterte.

Die breite Anwendung von Ultraschall in Krankenhäusern, Notfall- und Intensivstationen auch ohne genügende Weiterbildung machte es notwendig, auch für diese Kolleginnen und Kollegen eine adäquate Weiterbildung zu organisieren. Dazu gab es nach 2000 auch Entwicklungen in den USA (in früheren Jahren wusste man nicht viel über die dortige Sonografie) mit dem sog. «Point of Care»-Konzept, das viele Klinikerinnen und Kliniker in Europa begeisterte [7]. «Point of Care Ultrasound» oder POCUS wird mit Erfolg auch in Afrika zu spezifischen Fragestellungen angewendet [8].

Das EPSC (Education and Professional Standards Committee) hat bereits 2012 unter Leitung von J. Tuma [9] die wichtigsten Eckpfeiler des Ultraschallunterrichts skizziert.
Neben Bemühungen um Harmonisierung des Kurswesens in Europa wurden damals erste Schritte Richtung e-Learning gemacht, «EFSUMB-Learning»-Zentren gegründet (in der Schweiz: Zentrum Hirslanden, unter Leitung von A. Serra und J. Tuma) und Verschiebung des Ultraschallunterrichts an die Universitäten. Zu dem Zeitpunkt war die Entwicklung an einigen Universitäten in den USA bereits weiter [10]. Zusammen mit G. Mathis gelang es im EPSC, den Unterricht an europäischen Universitäten zu erfassen, was beim Dreiländertreffen 2013 in Stuttgart präsentiert wurde [11]. Dank einer Initiative in Wien (Prosch) hat man zusammen mit Studentinnen und Studenten das Konzept von «Sono4you» entwickelt, dabei lehren und lernen die Studierenden die Sonoanatomie selber. Sono4you Zürich wurde bereits 2015 gegründet [12]. 2017 wurde im Rahmen des Fortbildungskongresses ein Grundkurs mit Teilnahme der Studierenden organisiert. In der Folge wurde im Rahmen der SGUM-Sektion «Young Sonographers» das Konzept des «Blended Learning» entwickelt und in Koordination mit Schweizer Universitäten verwirklicht [13]. So ist es nun endlich so weit, dass Ultraschall neben dem Stethoskop ein integraler Bestandteil der Ausbildung von Ärztinnen und Ärzten sein wird.

Neben der Weiterentwicklung neuerer Techniken sowie dem POCUS-Konzept für breit abgestützte klinische Sonografie verschiebt sich zurzeit der Schwerpunkt der Grundausbildung an die Universitäten, und das ist gut so. Speziell zu diesem Thema siehe das Editorial von H.-R. Schwarzenbach.

\section{Bibliografie}

1. Seitz, KH: Gerhard Rettenmaiert: Pionier der internistischen Sonographie. Dtsch Arztebl 2016; 113:A-505/B-425/C-421.

2. Tuma J, Baugartner M, Moch H, Serra A: CME-Sonografie 89: Differenzialdiagnose von Nierenraumforderungen. Praxis 2020; 109: 71-77.

3. Weiss D: Möglichkeiten der kontrastunterstützten Ultraschalldiagnostik bei Erkrankungen des Gastrointestinaltrakts. Praxis 2020; 109: 615-630.

4. Mathis G: Lungenultraschall zur Differenzialdiagnose der Atemnot.Praxis 2020; 109: 592-595.

5. Gassmann B: Farbdopplersonografie: Geräteeinstellungen bedingen die Diagnosequalität und das Auftreten von Artefakten. Praxis 2020; 109: 566-571.

6. Häusermann M: Die Rolle der Sonografie in der Hausarztmedizin. Praxis 2020; 109: 596-607.

7. Moore L, Copel JA: Point of care ultrasonography. N Engl J Med 2011; 364:749-757.

8. Ngome O, Rohacek M: Point-of-Care Ultrasound: A Useful Diagnostic Tool in Africa. Praxis 2020; 109: 608-614. 
9. Tuma J: What is the EPSC? Ultraschall Med 2012; 33:189-190.

10. Hoppmann RA, Rao VV, Poston MB, et al.: An integrated ultrasound curriculum (iUSC) for medical students: 4-year experience.Crit Ultrasound J 2011; 3:1-12.

11. Mathis G, Blank V, Tuma J: EFSUMB- Survey on student ultrasound education. Stuttgart; Euroson: 2013.

12. Hangartner R, von Felten E, Arumuganathan N, Weber T: Sono4You Zürich\$. Praxis 2016; 105: 692-692.

13. Hari R, Meienberg A, Braun L: Ultraschallausbildung für Studierende in der Schweiz: Ein Consensus von Universitäten und

der SGUM. Schweiz Ärzteztg 2020; 101: 251-253.

\section{PD Dr. med. Jan Tuma}

Konsiliararzt für Sonographie

Klinik Hirslanden

Witellikerstrasse 40

8032 Zürich

jan.tuma@hin.ch 